Article

\title{
Spectral Domain Optical Coherence Tomography Imaging Performance Improvement Based on Field Curvature Aberration-Corrected Spectrometer
}

\author{
Seung Seok Lee ${ }^{1}$, Woosub Song ${ }^{2}$ and Eun Seo Choi ${ }^{1 \text {,* }}$ \\ 1 Department of Physics, Chosun University, Gwangju 61452, Korea; edc1532@chosun.ac.kr \\ 2 Medical Photonics Research Center, Korea Photonics Technology Institute, Gwangju 61007, Korea; \\ wsong@kopti.re.kr \\ * Correspondence: cesman@chosun.ac.kr
}

Received: 30 April 2020; Accepted: 22 May 2020; Published: 25 May 2020

Featured Application: SD-OCT imaging depth enhancement with improved contrast.

\begin{abstract}
We designed and fabricated a telecentric f-theta imaging lens (TFL) to improve the imaging performance of spectral domain optical coherence tomography (SD-OCT). By tailoring the field curvature aberration of the TFL, the flattened focal surface was well matched to the detector plane. Simulation results showed that the spot in the focal plane fitted well within a single pixel and the modulation transfer function at high spatial frequencies showed higher values compared with those of an achromatic doublet imaging lens, which are commonly used in SD-OCT spectrometers. The spectrometer using the TFL had an axial resolution of $7.8 \mu \mathrm{m}$, which was similar to the theoretical value of $6.2 \mu \mathrm{m}$. The spectrometer was constructed so that the achromatic doublet lens was replaced by the TFL. As a result, the SD-OCT imaging depth was improved by $13 \%(1.85 \mathrm{~mm})$ on a $10 \mathrm{~dB}$ basis in the roll-off curve and showed better sensitivity at the same depth. The SD-OCT images of a multi-layered tape and a human palm proved that the TFL was able to achieve deeper imaging depth and better contrast. This feature was seen very clearly in the depth profile of the image. SD-OCT imaging performance can be improved simply by changing the spectrometer's imaging lens. By optimizing the imaging lens, deeper SD-OCT imaging can be achieved with improved sensitivity.
\end{abstract}

Keywords: SD-OCT; imaging depth; roll-off; field curvature aberration; spectrometer

\section{Introduction}

Optical coherence tomography (OCT) is a high-resolution optical imaging modality that combines a wide spectral light source-based optical interferometer and a fast point scanning method. OCT finds the internal structure of living tissue by tracking the path of light reflected at the interface between materials with a different refractive index [1]. The OCT system, which is implemented based on the principle of the basic interferometer, was developed as an optical biopsy technique for non-invasive imaging of various internal diseases. OCT was first released for use in the ophthalmology field and has recently been used as a unique technique for imaging the inside of the retina. Because of its high axial resolution, OCT can also be used for the early diagnosis of diseases as it can acquire tomographic images of easily accessible skin tissues and can also detect diseases occurring in the airways, stomach, blood vessels and urethra by using optical probes developed in the form of catheters [2-5]. Thus, OCT is one of the latest optical measurement techniques and it has recently been developed as a diagnostic technique that is capable of providing multiple information beyond simple non-invasive tomographic images [6-9]. 
The first-generation OCT measured interference fringes over the measurement time [10]. The optical path of the reference arm is changed over time, and the difference in the optical path of the sample arm is used to identify the boundary in a sample by finding the maximum position from the visibility envelope of the interference signal. In the time-domain measurement, the location of the internal boundary of the sample is detected by coherence gating while periodically changing the light path in the reference arm. Because coherence gating generates interference fringes only when the optical path length difference (OPD) is within a coherence length, the optical path has to be mechanically changed to remain within the coherence length. However, physically changing the optical path at a high rate of repetition has been a major limiting factor in implementing high-speed real-time OCT systems.

Several improved methods have been proposed, and a repetition rate of tens of $\mathrm{Hz}$ can be achieved [11]. However, despite these improvements there were still some limitations in performing real-time tomographic image-based diagnosis. In principle, the movement of the reference mirror produces additional frequency components in the fringe due to the Doppler effect. Therefore, the first-generation OCT system required a bandpass filter with a wide frequency bandwidth to filter the interference signal without information loss, which is accompanied by the problem of increasing noise. Even when the optimum bandpass filter was designed, the fundamental problem of high background noise remained. When coherence gating was not applied, non-interfering signals for all wavelengths of a broadband light source were measured through a bandpass filter. To effectively address the limitations of the first-generation OCT, a different detection method was needed to obtain the same information without mechanically changing the OPD for the coherence gating. OCT that uses the new approach is known as the second-generation OCT or Fourier-domain OCT.

Second-generation OCT does not measure the interfering signal in the time domain but in the frequency domain [12]. In a given OPD, the interference efficiency according to wavelength is not the same and exhibits periodic characteristics. When the intensity of each wavelength component is measured while the optical path of the reference arm is fixed, the change in visibility in the form of a sinusoidal function is measured. The periodicity contained in the measurement signal can be extracted by using Fourier transformation [13]. This process provides site information about the sample's internal boundaries. This information allows the non-invasive reconstruction of tomographic images of samples. Because the interference signal in the second-generation OCT is measured in the spectral domain not in the time domain, the background noise can be effectively reduced, unlike first-generation OCT where all of the wavelength components of the broadband light source are measured simultaneously. Additionally, the second-generation OCT greatly improves the speed of image acquisition with the help of a fast wavelength tuning method or high-speed line scan camera [14,15].

To perform Fourier-domain OCT, a conventional fiber optic interferometer utilizes a wavelength-tunable light source instead of the broadband light source. The output wavelength of the wavelength-tunable light source is periodically and repeatedly changed. The light intensity of each wavelength changes due to the difference in interference efficiency for the wavelength after passing through the interferometer. Therefore, the output signal of the interferometer is interpreted as an interference signal according to the wavelength component [16]. This method distinguishes wavelength components over sweeping time and measures spectral interference. The intraocular distance of the eye was successfully measured using this method [17]. Another method is to use a spectrometer consisting of a linear detector array. This method measures the interference signal at a time for each wavelength component [18]. The former method is called swept-source OCT (SS-OCT) and the latter is called spectral domain OCT (SD-OCT). SS-OCT measures a fringe signal by using a single detector and a light source capable of wavelength tuning. SD-OCT, on the other hand, consists of the broadband light source and the spectrometer. SS-OCT has been able to achieve repetition rates of several $\mathrm{MHz}$ owing to the continuous increase in wavelength sweeping speed [19]. An increase in the repetition rate provides the advantage of obtaining a clear image that is independent of the movement of the biological tissue and also enables the realization of a high-speed $4 \mathrm{D}$ image $[20,21]$. 
However, the wavelength-swept light source based on a microelectromechanical systems (MEMS) actuator has a problem in that the fringe signal sometimes appears at an OPD that is larger than the coherence length of the light source. Because the variable wavelength is mechanically driven, the phase in SS-OCT is unstable. As an alternative to addressing unstable phase performance, an akinetic wavelength sweeping source has been developed and applied to various OCT applications [22]. SS-OCT systems with high repetition rate adjustable light sources require high-speed digitizers for system configuration. Such a system configuration has a high-cost because the implementation and control measures for very sophisticated signal measurement must be applied to avoid image distortion. In contrast, SD-OCT is based on a broadband light source, so the cost of implementation is lower than that of SS-OCT. SD-OCT generally has the advantage of superior phase stability over wavelength tuning-based measurement because it typically requires no moving device. However, the SD-OCT system requires both a high-speed linear array-based spectrometer and a high-speed signal acquisition image grabber. Recently, due to the development of a USB-based high-speed spectrometer, high-speed measurements can be achieved without any image grabber. When configuring the system at 1300 $\mathrm{nm}$ spectral windows, the linear detector array is very expensive, which can be a major obstacle in the implementation at this spectral window. Nevertheless, the SD-OCT system has the advantage of simpler system implementation and easier operation than the SS-OCT system.

The SD-OCT system consists of a light source, interferometer, and spectrometer. As mentioned above, the light source is usually a broadband light source such as LED, superluminescent diodes (SLD), or a supercontinuum source. The interferometer may be composed of optical fibers or bulk optics if necessary. It is common to use an interferometer in the Michelson or Mach-Zehnder configuration. The spectrometer is the heart of the SD-OCT system. Signal acquisition at high speed is performed in the spectrometer, however, distortion of a fringe signal can occur. For that reason, research on SD-OCT has been focused on the development of an optimized spectrometer for measuring interfering signals without signal distortion and extending the imaging depth.

Several methods have been proposed to improve the ranging depth of SD-OCT. First, a method for improving the optical system in the sample arm can be considered. The depth of focus can be improved by using the Bessel beam of an axicon lens [23-25]. However, because only limited lenses can be used for this method, a signal processing-based approach has been suggested. For example, a study using a wavefront shaping technique, a method for correcting aberration, has been published [26]. A bidirectional imaging scan in the sample arm method was reported for overcoming limited image depth [27]. A full ranging method based on a phase-shifting technique doubled the depth of an image by generating complex signals from multiple images [28,29].

A method for improving the image depth in the SD-OCT using a spectrometer with a fixed number of pixels is proposed in this study. To increase the depth of the image, we need to improve the resolution of the spectrometer by increasing the number of pixels. Although a spectrometer with a fixed number of pixels is used, the pixel shift effect of moving the spectrometer can effectively increase the number of pixels by increasing the number of samples [30]. The disadvantage of this technique is that the imaging speed is limited due to the use of mechanical movement. The use of an optical frequency comb can improve the spectrum sampling function and extend image depth [31]. However, this method requires the use of a specialized device, an optical frequency comb generator, and sophisticated system control. Recently, a method for optically performing wave number linearization using a prism was used in a spectrometer [32,33]. Although the method improved the imaging depth, the optical alignment was more difficult.

The issue of the image depth of the SD-OCT is due to the spectrometer itself; thus, the ultimate solution is to supplement the spectrometer's performance by developing an optical design with improved properties. Among the optical elements that constitute the spectrometer, the imaging lens has different optical aberration characteristics depending on the optical design, which greatly affect the distortion of the detected interference signal. The fringe signal generated by the interferometer is measured by the linear detector array in the spectrometer. An imaging lens is inserted in front of the 
linear detector array to focus the dispersed light beam into the pixels. The imaging lens has a light focusing function, but a spherical focal surface is formed due to the shape of the lens. This curved surface is inconsistent with the plane of the pixel measuring light intensity, resulting in a decrease in the light-receiving efficiency. The visibility of the fringe signal measured in the planar detector array is reduced depending on the aberration characteristics of the imaging lens. This problem has been mentioned in a qualitative sense in previous research [34]. The unmatched focal surface restricts the imaging depth that can be achieved in SD-OCT. However, few studies have suggested good solutions to these problems.

In this study, we designed an imaging lens with improved field curvature aberration. Spectrometers were developed using imaging lenses with different field curvature aberrations, and the differences in imaging performance due to focal plane mismatch with a planar detector array were compared. We confirmed that improving the spectrometer's performance by reducing the field curvature aberration of the imaging lens helps to increase the contrast and ranging depth of the OCT image.

\section{Spectrometer Design Process}

We intend to show that simply replacing the imaging lens with improved aberration characteristics can significantly improve the imaging performance of the SD-OCT system. Because the performance of the spectrometer varies greatly according to the characteristics of the optical elements, the same optical devices were applied to each spectrometer except for the imaging lens. The imaging lenses that were utilized in the spectrometers were an achromatic doublet lens (ADL, AC254-060-B-ML, THORLABS), which is commonly used in spectrometer development, and a telecentric $\mathrm{f}$-theta lens (TFL) designed to reduce field curvature aberration. To design a spectrometer that is easy to implement, most of the optical components used were commercial products, and a simple optical configuration was selected.

The optical design of the spectrometer was done according to the following procedure [35]. The single-mode fiber connected to the spectrometer has a core size of about $6 \mu \mathrm{m}$ and the dimensions of a single-pixel of a line-scan camera (LSC, 2048 pixels, CMOS sensor, 140 kHz, SPL2048-140 km, Basler), that is, $10 \mu \mathrm{m} \times 10 \mu \mathrm{m}$. Therefore, the amplification of the spectrometer will be less than 1.67 to avoid intensity loss and cross-talk between pixels. Because the amplification of the spectrometer is given by the ratio of the focal length of the collimator and the imaging lens, the focal length of the imaging lens in front of the LSC can be determined from the amplification value. When we consider that the focal length of the collimator (F810APC-842, THORLABS) is about $36.2 \mathrm{~mm}$, the focal length of the imaging lens is calculated as about $60 \mathrm{~mm}$. The collimated beam with a diameter of $7.8 \mathrm{~mm}$ from the fiber-optic collimator is directed to a transmission grating (1800 lines $/ \mathrm{mm}$, a center wavelength of 840 $\mathrm{nm}$, WASATCHPHOTONICS) at an incident angle of $49.1^{\circ}$. After passing through the transmission gratings, the spectral components are diverged according to the diffraction grating equation. To satisfy the Nyquist sampling theory, the total spectral bandwidth received by the spectrometer must be at least twice the full width at half maximum (FWHM) spectral bandwidth of the light source [36]. For this reason, each edge wavelength is designed to be $778 \mathrm{~nm}$ and $900 \mathrm{~nm}$, respectively. The calculated diffraction angle at each edge wavelength is $40.13^{\circ}$ and $59.77^{\circ}$. At the center wavelength of $840 \mathrm{~nm}$, the diffraction angle is $49.11^{\circ}$. This results in a divergence angle of $19.64^{\circ}$ for the designed full spectral bands. This value is also a critical factor in determining the focal length of the imaging lens. When the light beam is incident on a lens with a focal length $F$ at an angle of $\theta$, the height of the image at the focal position is the product of $F$ and $\theta$ by the ABCD matrix method. As shown in Figure 1, when this is the case, the relation of $\Delta \varphi \times f_{2}=L_{\mathrm{c}}$ must be satisfied. If the length of the active area of the LSC is $20.48 \mathrm{~mm}$ and the divergence angle after the transmission grating is about $19.7^{\circ}$, then the focal length is calculated as $59.7 \mathrm{~mm}$. We can confirm that this value is in good agreement with the value obtained by considering the amplification of the spectrometer. 


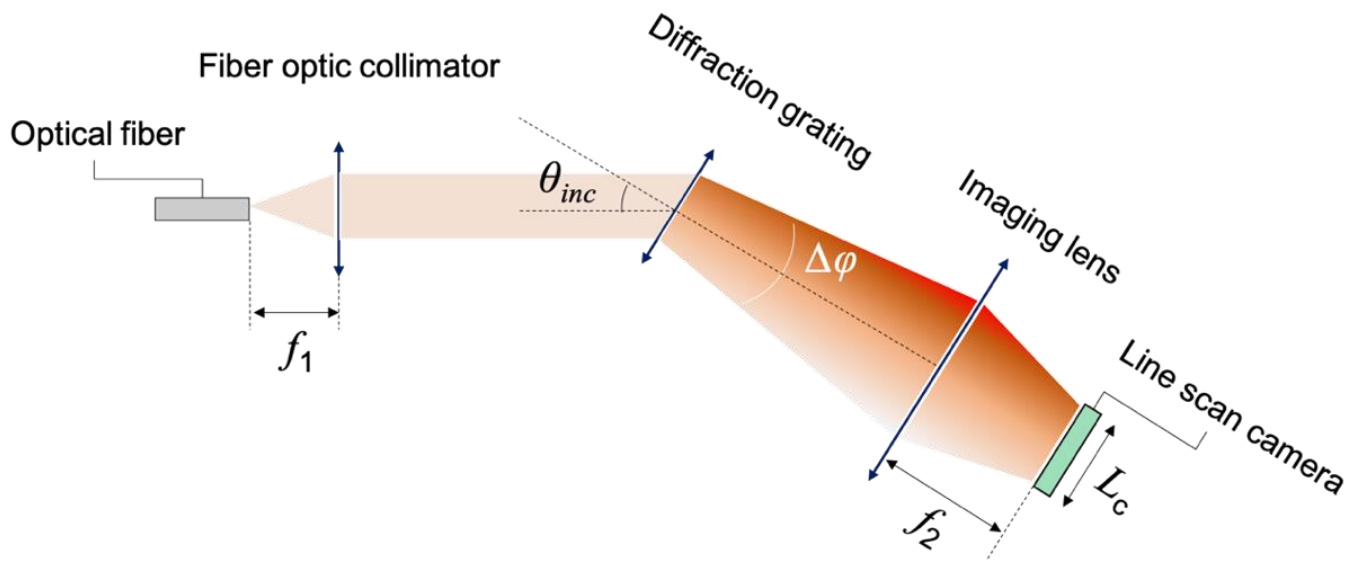

Figure 1. Spectrometer design parameters. The focal length of the fiber-optic collimator is $f_{1}$, the incident angle to the transmission gratings is $\theta_{\text {inc, }}$ the diffracted divergence angle is $\Delta \varphi$, the focal length of the imaging lens is $f_{2}$, and the length of the line-scan camera (LSC) is $L_{c}$.

Most existing telecentric f-theta lenses are designed for laser processing using high power lasers with a wavelength of $1060 \mathrm{~nm}$ or designed for vision application with visible light. However, it is not appropriate to use this lens because the light source considered in this experiment is not a laser, but a broadband light source with a wide wavelength band of several tens of $\mathrm{nm}$ centered around $840 \mathrm{~nm}$. Also, although scan lenses for OCT have been developed and are commercially available, we had to design and fabricate new imaging lenses because various specifications such as the lens size, the field of view, focal length, aberration characteristics and so on are not suitable for use in the spectrometer designed in this experiment. Correcting the aberration of an existing lens using multiple commercial lenses is difficult due to the different aberration effects of each lens. In addition, the use of multiple lenses increases the light loss and volume of the system. These problems limit the development of a simplified spectrometer.

The TFL was designed taking into account the spectrometer design parameters. The TFL consisted of 4 lenses made of Lanthanum glass, which has a high refractive index and is useful for correcting the chromatic and spherical aberration of the lens. The clear aperture of the first lens was $32 \mathrm{~mm}$ in diameter and the last lens was $22 \mathrm{~mm}$. This value was intended to ensure that the light at the edge wavelength avoids cutting at the edge of the lens. The angle of incidence for the TFL was designed to be 34.2 degrees to correspond to the divergence angle of the $200 \mathrm{~nm}$ spectral bandwidths to accommodate the entire divergent beam, which corresponds to the total spectral bandwidth of $120 \mathrm{~nm}$ considered in the spectrometer design. The effective focal length was $60 \mathrm{~mm}$, the focal length of the spectrometer design parameter. The scan length was $31 \mathrm{~mm}$, corresponding to the $200 \mathrm{~nm}$ spectral bandwidths. The value was larger than the length of the LSC. The length of the TFL was $41 \mathrm{~mm}$.

\section{Aberration Simulation of Each Imaging Lens}

Before developing the spectrometer, aberration characteristics were simulated using ZEMAX to understand the characteristics of the two imaging lenses, an ADL and a TFL. The ADL was originally designed to reduce spherical and chromatic aberrations and is commonly used as an imaging lens for spectrometers. However, in the ADL, the focal surface is formed as a curved shape. To address this problem, the TFL was designed to have a flat focal plane and significantly reduce the distortion of the focal surface.

Among the lens aberrations, simulations for field curvature and astigmatism were performed over full spectral bands, and the results can be seen in Figure 2. As the angle increased, the tangential and sagittal focal surface of the ADL became further away from the flat image plane, resulting in a maximum shifting of $-1.2 \mathrm{~mm}$ and $-0.5 \mathrm{~mm}$ for the tangential and sagittal ray, as shown in Figure 2a. The maximum difference between the two focal positions was $0.7 \mathrm{~mm}$. The TFL showed improved field 
curvature and astigmatic aberration characteristics compared to the ADL. The simulation results for the TFL are shown in Figure 2b, where the tangential focal position was shifted up to $0.28 \mathrm{~mm}$, and the sagittal focal position was moved up to $-0.12 \mathrm{~mm}$. Their maximum difference was $0.4 \mathrm{~mm}$. When we considered that the FWHM spectral bandwidths correspond to an incident angle of around 4 degrees, the maximum focal shifts occurred in the tangential ray and were about $270 \mu \mathrm{m}$ for the ADL and about $140 \mu \mathrm{m}$ for the TFL. The minimum shifts were about $130 \mu \mathrm{m}$ for the ADL and about $85 \mu \mathrm{m}$ for the TFL in the sagittal ray. The TFL presented a nearly flat focal surface similar to the detector plane. The astigmatism characteristics of each imaging lens did not change significantly with wavelength, and as a result, we can confirm that the chromatic aberration was well corrected for both imaging lenses.

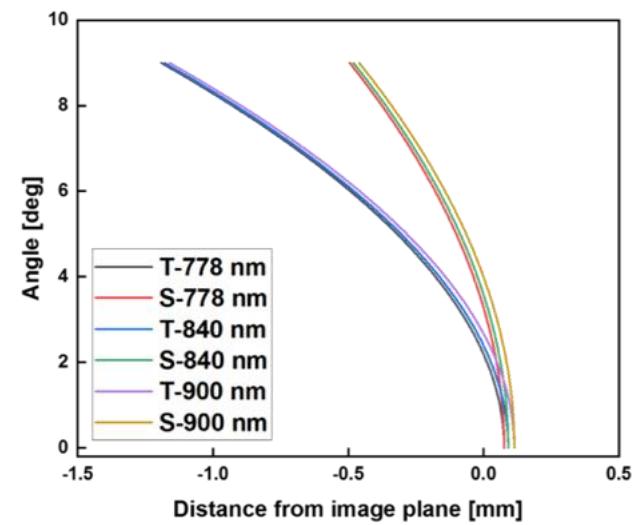

(a)

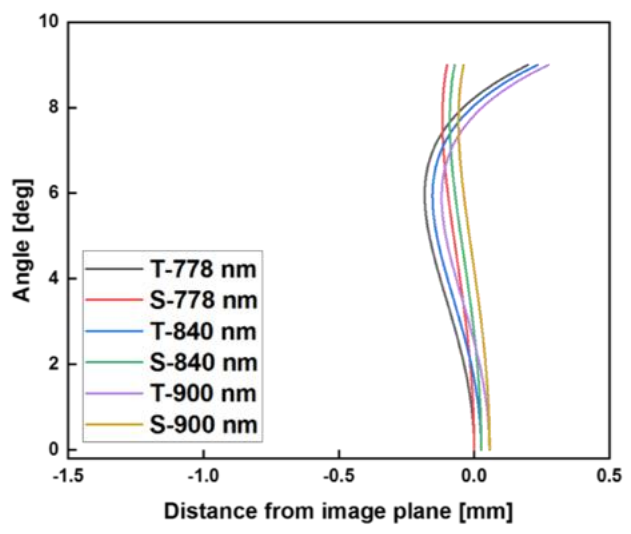

(b)

Figure 2. The field curvature and astigmatic aberration characteristics of each imaging lens. T and S mean the tangential and sagittal focal position of (a) the achromatic doublet lens (ADL) and (b) the telecentric f-theta lens (TFL).

If the focal position is not placed on the pixel surface, the visibility of the detected spectral interference signal is poor. In the simulation results, the TFL showed better field curvature than the ADL. Therefore, we can expect that the spectrometer using the TFL will measure interference signals with enhanced visibility and an OCT image obtained with this spectrometer will have better image contrast.

When the focal length is different depending on the wavelength in the same lens, this axial aberration is called the chromatic focal shift. Simulations for the chromatic aberration of the two imaging lenses were also performed and the results were calculated for the entire spectral bandwidths, as shown in Figure 3. The ADL showed a focal shift of less than $40 \mu \mathrm{m}$ and the TFL had a focal shift of less than $60 \mu \mathrm{m}$. With regard to the FWHM spectral bandwidths, the chromatic focal shifts were less than $15 \mu \mathrm{m}$ and $20 \mu \mathrm{m}$, respectively. The ADL was found to have a smaller chromatic focal shift than the TFL. However, the two imaging lenses had similar values. Both imaging lenses showed nearly uniform chromatic focal length, indicating that chromatic aberration was well corrected in both lenses. The chromatic aberration characteristics of these two imaging lenses were in good agreement with the results shown in Figure 2, whereby the change in the focal position change according to the wavelengths was very small. 


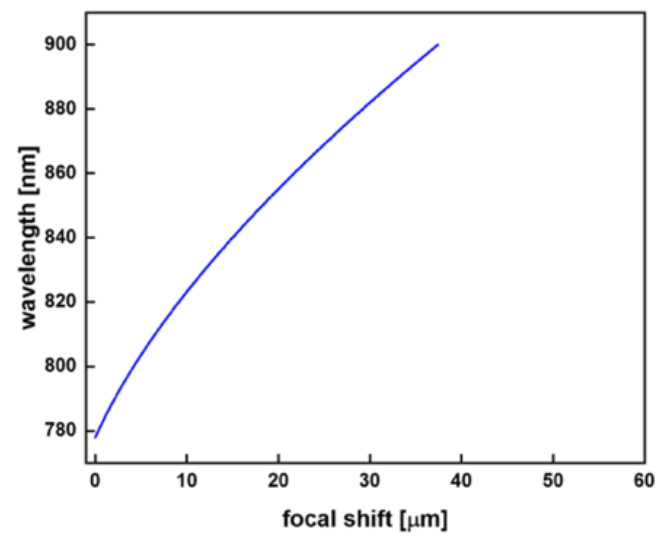

(a)

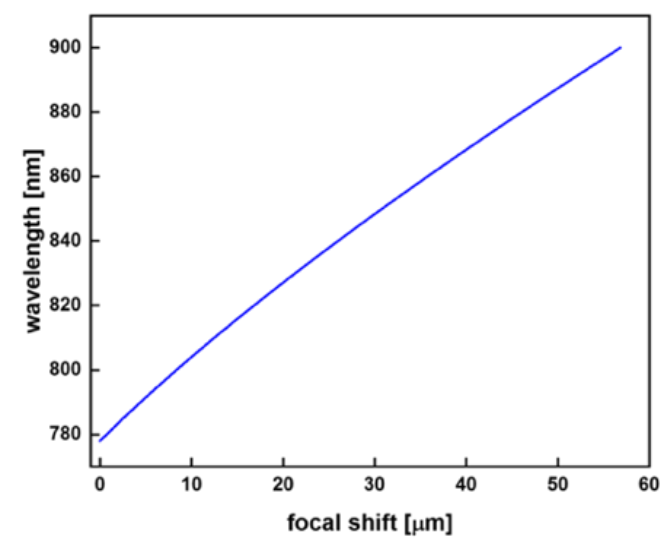

(b)

Figure 3. Chromatic focal shift calculated for (a) the ADL and (b) the TFL.

In the following simulation, the spot size of the beam in the detector plane was calculated. The spot diagrams were displayed in Figures 4 and 5 . For the ADL, the root mean square (RMS) spot radius is $\sim 3 \mu \mathrm{m}$ at the central wavelength and extended to $\sim 20 \mu \mathrm{m}$ at the edge wavelength of the FWHM spectral bandwidth, as seen in Figure 4. However, as seen in Figure 5, the RMS spot radius of the TFL was less than $2 \mu \mathrm{m}$ at the center wavelength and increased to $\sim 5 \mathrm{~m}$ at the edge wavelength. Because the length of the single pixel is $10 \mu \mathrm{m}$, the spectrometer with the ADL may experience intensity loss and spectral crosstalk. Unlike the TFL, the ADL causes fringe visibility degradation at wavelengths shorter or longer than the central wavelength. The scale for the spot diagram in Figures 4 and 5 was $100 \mu \mathrm{m}$ and $20 \mu \mathrm{m}$, respectively.

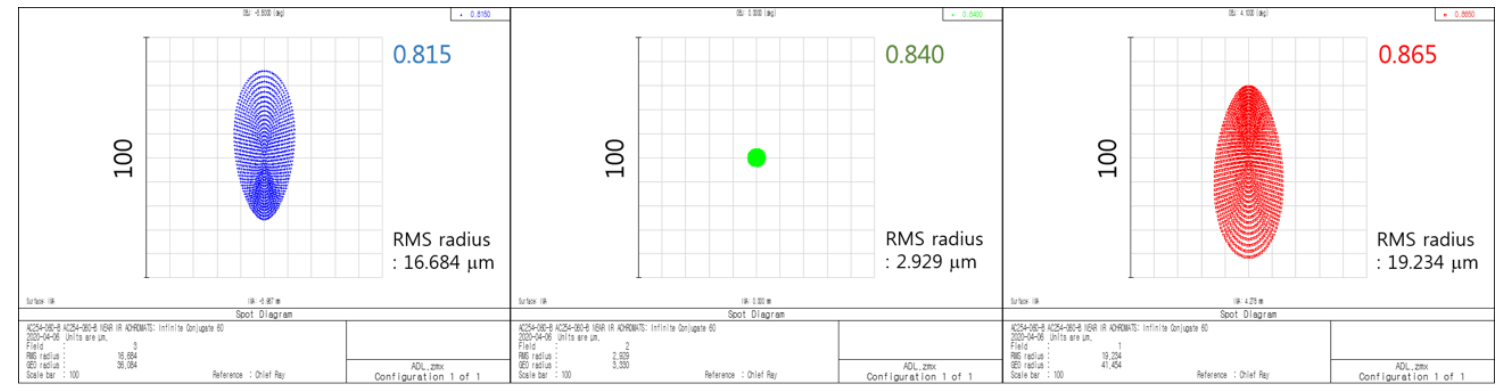

(a)

(b)

Figure 4. Spot diagram of the ADL at (a) the edge wavelength of $815 \mathrm{~nm}$, (b) the center wavelength of $840 \mathrm{~nm}$, and (c) the edge wavelength of $865 \mathrm{~nm}$. The plot scale is $100 \mu \mathrm{m}$. The number on the right top is the wavelength used for calculation.

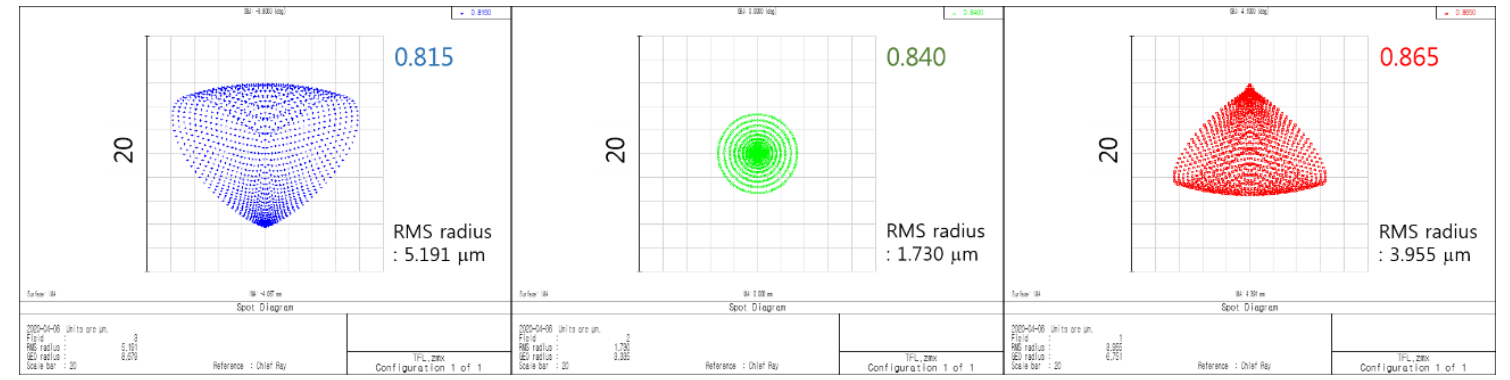

(a)

(b)

(c)

Figure 5. Spot diagram of the TFL at (a) the edge wavelength of $815 \mathrm{~nm},(\mathbf{b})$ the center wavelength of $840 \mathrm{~nm}$, and (c) the edge wavelength of $865 \mathrm{~nm}$. The plot scale is $20 \mu \mathrm{m}$. The number on the right top is the wavelength used for calculation. 
The modulation transfer function (MTF) curve as a function of spatial frequency is given in Figure 6. The MTF curve helps us to understand the contrast characteristics of the imaging lenses. High MTF values at high spatial frequencies indicate that the SD-OCT system is able to provide bright, high-contrast imaging at deep depths. When looking at the MTF curve calculated at the three different incident angles, which correspond to the center and each edge wavelength, the two imaging lenses showed significant differences as the spatial frequency increased. The TFL presented a similar decreasing trend, regardless of incident angles, and maintained a similar MTF value at the maximum spatial frequency. However, the ADL provided very different curves depending on the incident angles and finally reached almost zero at the maximum spatial frequency. Using the TFL for the spectrometer could improve the achievable image depth and contrast. The MTF value for the TFL was between 0.4 and 0.6 at the Nyquist frequency of the LSC (50 lines/mm).

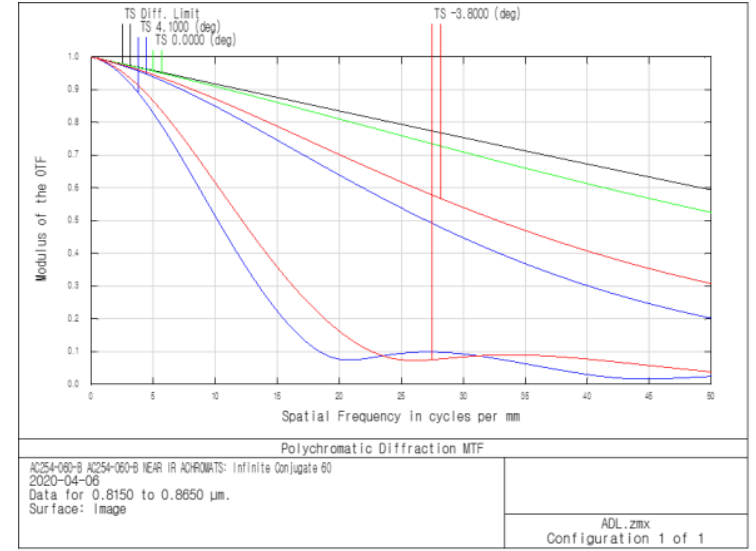

(a)

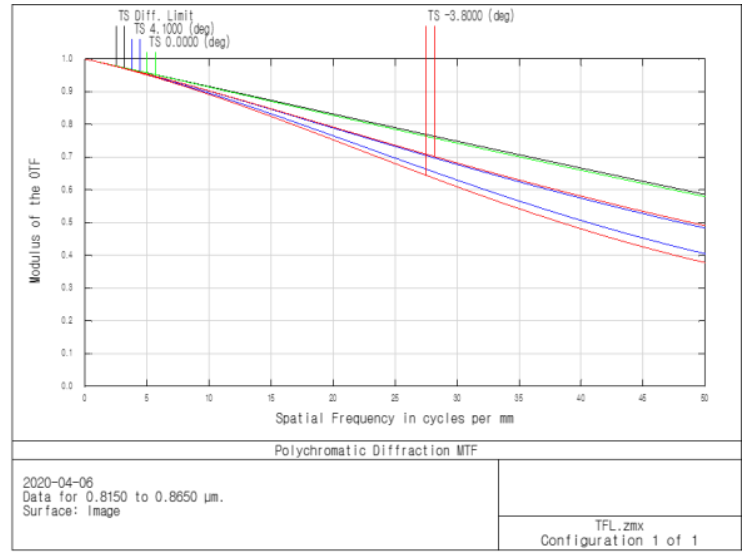

(b)

Figure 6. The modulation transfer function (MTF) curve for (a) the ADL and (b) the TFL at three incident angles of 0 degrees, -3 degrees, and 4 degrees corresponding to three wavelengths of $815 \mathrm{~nm}$, $840 \mathrm{~nm}, 865 \mathrm{~nm}$, respectively.

\section{Experimental Setup}

The light source used in the experiment was SLD (EXS210090-01, EXALOS), which had a central wavelength of $840 \mathrm{~nm}$, a spectral bandwidth of $50 \mathrm{~nm}$, and an output power of $12 \mathrm{~mW}$. The light beam from the light source was directed to a $2 \times 2$ fiber optic coupler that acts as fiber-optic Michelson interferometer and is split into a reference and sample arm. The sample arm consists of a fiber-optic collimator (F810APC-842, THORLABS), an achromatic doublet lens (AC254-040-B-ML, THORLABS) and a 2-axis Galvano-scanner to perform lateral scanning over a sample. The same devices, except for the 2-axis Galvano-scanner, were used in the reference arm to match the dispersion balance. The reflected light beams from both arms were recoupled to the fiber-optic collimator and generated interference signals depending on the OPD between two arms. The interference signal is propagated through single-mode fiber to a spectrometer, where the interference signal of 2048 data points was recorded by a high-speed frame grabber (NI PCI-e 1433, NATIONAL INSTRUMENTS) as a function of the spectral components of the SLD. After removing background noise and applying the Hanning window, the spectral signal was subjected to k-linearization to convert it as a function of wavenumber. For k-linearization, a wavenumber mapping table for each wavelength was prepared prior to the experiment using five fiber Bragg gratings. The interference signal after k-linearization was subjected to a discrete Fourier transform. OCT images were reconstructed by mapping Fourier transformed 1024 data on a grayscale. A program developed using LABVIEW was used for the system operation and signal processing. 


\section{Experimental Results}

\subsection{Spectrum Measurement Using the Implemented Spectrometers}

The characteristics of the spectrum measured by the two spectrometers were compared and analyzed. The measured spectra are shown in Figure 7. The spectrum measured with the spectrometer developed using the ADL (S-ADL) is shown in Figure 7a, and the spectrum measured with the spectrometer using the TFL (S-TFL) is shown in Figure $7 \mathrm{~b}$. To compare the spectral shape of the spectrum, each spectrum was normalized. Because the two spectrometers use the same LSC, different spectral shapes might be due to the imaging lens in the spectrometer. The spectra measured by the two spectrometers were similar to each other, and both FHWM bandwidths were equal to $42 \mathrm{~nm}$.

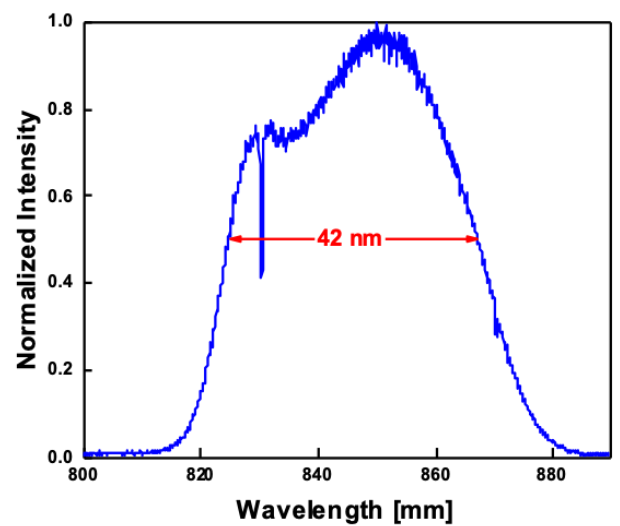

(a)

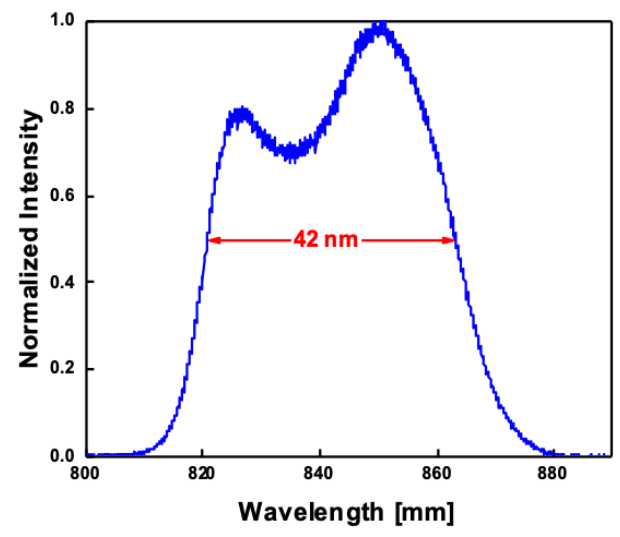

(b)

Figure 7. Spectrum measured by (a) the S-ADL, and (b) the S-TFL.

\subsection{Axial Resolution Performance Depending on the Spectrometer}

We measured the point spread function (PSF) using a flat mirror at the same position of $\sim 210$ $\mu \mathrm{m}$ in OPD to calculate axial resolutions. The results seen in Figure 8 showed the same values of $7.8 \mu \mathrm{m}$, which was in good agreement with the theoretical axial resolution of $6.2 \mu \mathrm{m}$. The variation may be due to the imbalance of dispersion in the interferometer. Although the aberration effect of the imaging lens was different, the difference in the axial resolution due to the aberration effect could not be distinguished and similar spectra were obtained by the two spectrometers. If we used a light source with larger spectral bandwidths, the difference in the axial resolutions due to imaging lens aberration may become more apparent.

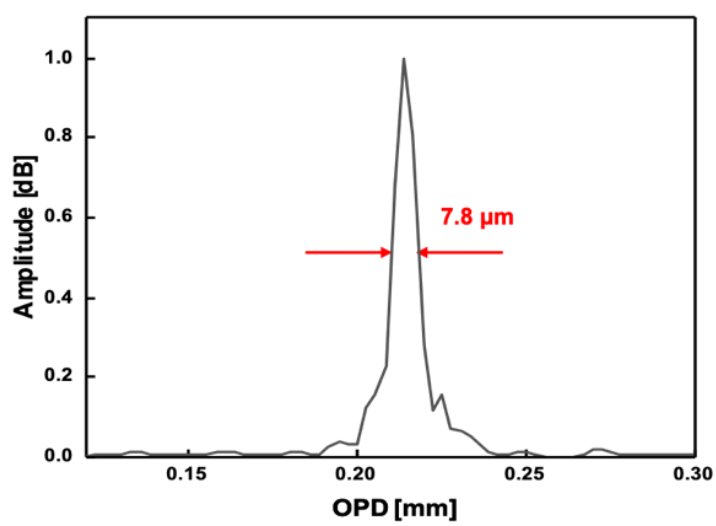

(a)

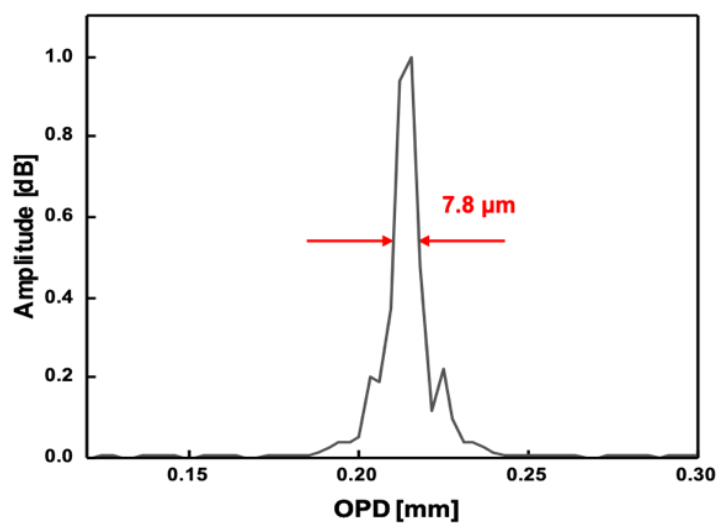

(b)

Figure 8. Axial resolution calculated from the point spread function (PSF) measured by (a) the S-ADL and (b) the S-TFL according to the optical path length difference (OPD). 


\subsection{Point Spread Function Performance According to the Spectrometer}

The difference in SD-OCT imaging performance due to the aberration of the imaging lens was more pronounced in roll-off performance than in the axial resolution. The maximum image depth was about $2.8 \mathrm{~mm}$ for the S-ADL and $3 \mathrm{~mm}$ for the S-TFL. The distance per single fast Fourier transform (FFT) bin was calculated using a gauge block and each spectrometer had values of $2.8 \mu \mathrm{m} / \mathrm{bin}$ and $3.0 \mu \mathrm{m} / \mathrm{bin}$. Because of the telecentric nature of the TFL, the spectrum in the S-TFL may be measured over a relatively larger number of pixels than that of the S-ADL, which has a curved focal plane. A series of PSFs were measured at $300 \mu \mathrm{m}$ intervals up to $3 \mathrm{~mm}$ and the results are shown in Figure 9 . Normalization was based on the maximum amplitude among the PSFs. At the OPD at $2.5 \mathrm{~mm}$, the axial resolutions for the S-ADL and the S-TFL were $12 \mu \mathrm{m}$ and $7.9 \mu \mathrm{m}$, respectively. The S-TFL maintained better axial resolution than S-ADL at the deep imaging depth.

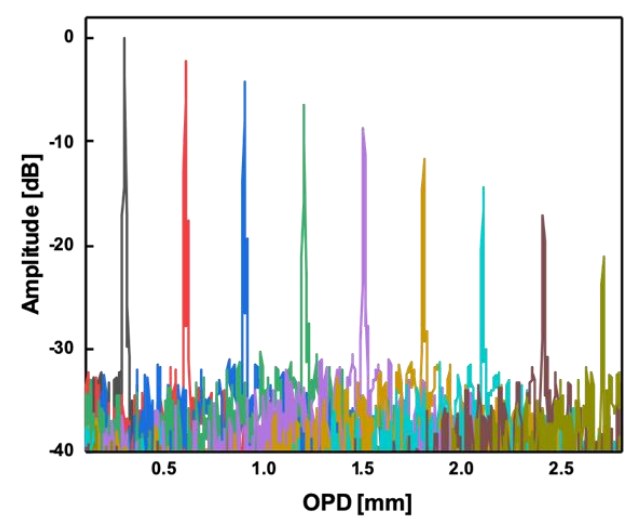

(a)

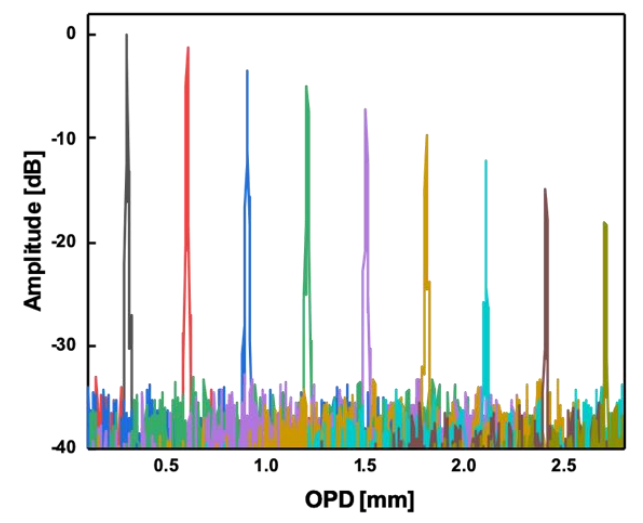

(b)

Figure 9. PSF measurement results according to OPD. PSFs obtained using (a) the S-ADL and (b) the S-TFL

\subsection{Sensitivity Roll-Off Characterization}

The sensitivity roll-off in Figure 10 was calculated from the measured PSFs. The sensitivity roll-off of the SD-OCT system using the S-TFL was better than that of the S-ADL. The SD-OCT system using the S-ADL had an imaging depth of $0.75 \mathrm{~mm}$ at a sensitivity roll-off of $-3 \mathrm{~dB}$, and the S-TFL-based SD-OCT systems had an imaging depth of $0.89 \mathrm{~mm}$ at the same sensitivity roll-off. At a sensitivity roll-off of $-6 \mathrm{~dB}$, the imaging depth was extended to $1.16 \mathrm{~mm}$ for the S-ADL and $1.33 \mathrm{~mm}$ for the S-TFL. When we considered a sensitivity roll-off of $-10 \mathrm{~dB}$, we found imaging depths of $1.63 \mathrm{~mm}$ and 1.85 $\mathrm{mm}$. Considering the results of the roll-off measurement, we can confirm that the aberration correction of the imaging lens was a critical factor in increasing the imaging depth of the SD-OCT system.

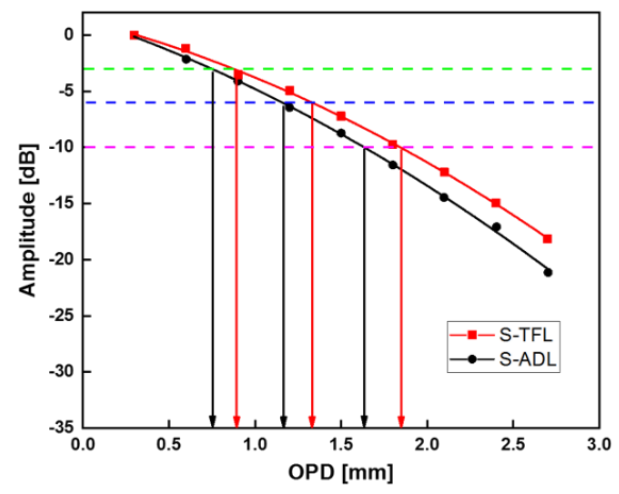

Figure 10. Sensitivity roll-off of the spectral domain optical coherence tomography (SD-OCT) system according to the OPD when using the S-ADL and the S-TFL. 


\subsection{SD-OCT Imaging Performance}

SD-OCT images of non-biological samples and biological samples were obtained to verify the differences in the images resulting from improved imaging lens characteristics. As a non-biological sample, OCT images of tape with multiple layers were acquired. Two OCT images are displayed in Figure 11. Each OCT image is composed of 1024 (in the axial direction) by 500 (in the lateral direction, $2.55 \mathrm{~mm}$ ) pixels. By comparing the images in Figure 11a,b, it can be seen that the imaging depth of the OCT image acquired with the S-TFL was deeper than that of S-ADL, as confirmed by the roll-off characteristic. Three horizontal guidelines are drawn in Figure 11 for easy comparison of the image depths. At the third line from the top, the boundary of the tape is more clearly distinguished in Figure 11a compared to Figure 11b. For a more detailed comparison, depth profiles were extracted for the same A-line of each image. The depth profile for the S-TFL has a greater amplitude than the S-ADL. Even at deep depths, this feature was well preserved, as shown in Figure 11c. We already compared the curve of the MTF for each imaging lens after we obtained the simulation results. The MTF value is related to the amount of light that can be collected through the lens. Although the OCT image was obtained under the same conditions, Figure $11 \mathrm{~b}$ shows a brighter image and improved contrast at a deep depth than Figure 11a. This result reflects the characteristics of the imaging lens, therefore, TFL which has a higher MTF value than the ADL produces better image contrast and depth. This characteristic was also confirmed when a biological sample was used.
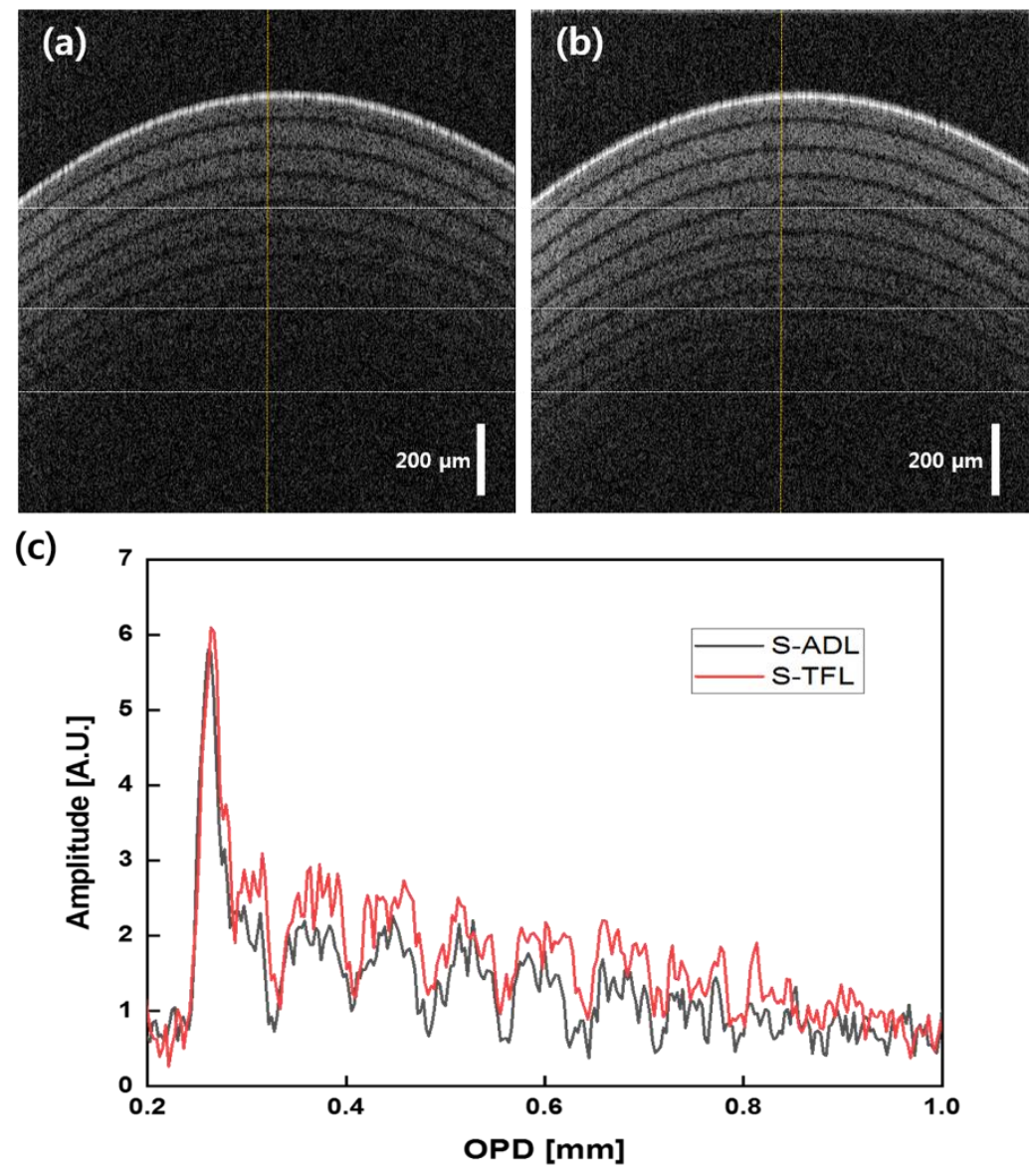

Figure 11. OCT image of a tape obtained using (a) S-ADL and (b) S-TFL. (c) Depth profiles at the same A-line of each image.

Differences in the brightness and the imaging depth were also distinguished in the OCT image of a human palm. The OCT image shown in Figure 12a was obtained with the S-ADL and has low contrast, while the image in Figure 12b obtained with the S-TFL shows a well-defined boundary inside 
the tissue, even at a deep depth. As in Figure 11a,b, three guidelines are drawn in the horizontal direction. Looking at the second guideline from the top, the image obtained by the S-ADL was not distinguishable, and as the depth increased, the interface inside the tissue was not visible. However, at the same depth, we can see that the interface is more distinct in Figure 12b than in Figure 12a. This difference can be explained more clearly through a specific A-line profile. When looking at depth profiles extracted from the same location in each image, the depth profile obtained for the S-TFL maintained a higher amplitude along the depth than that for the S-ADL.
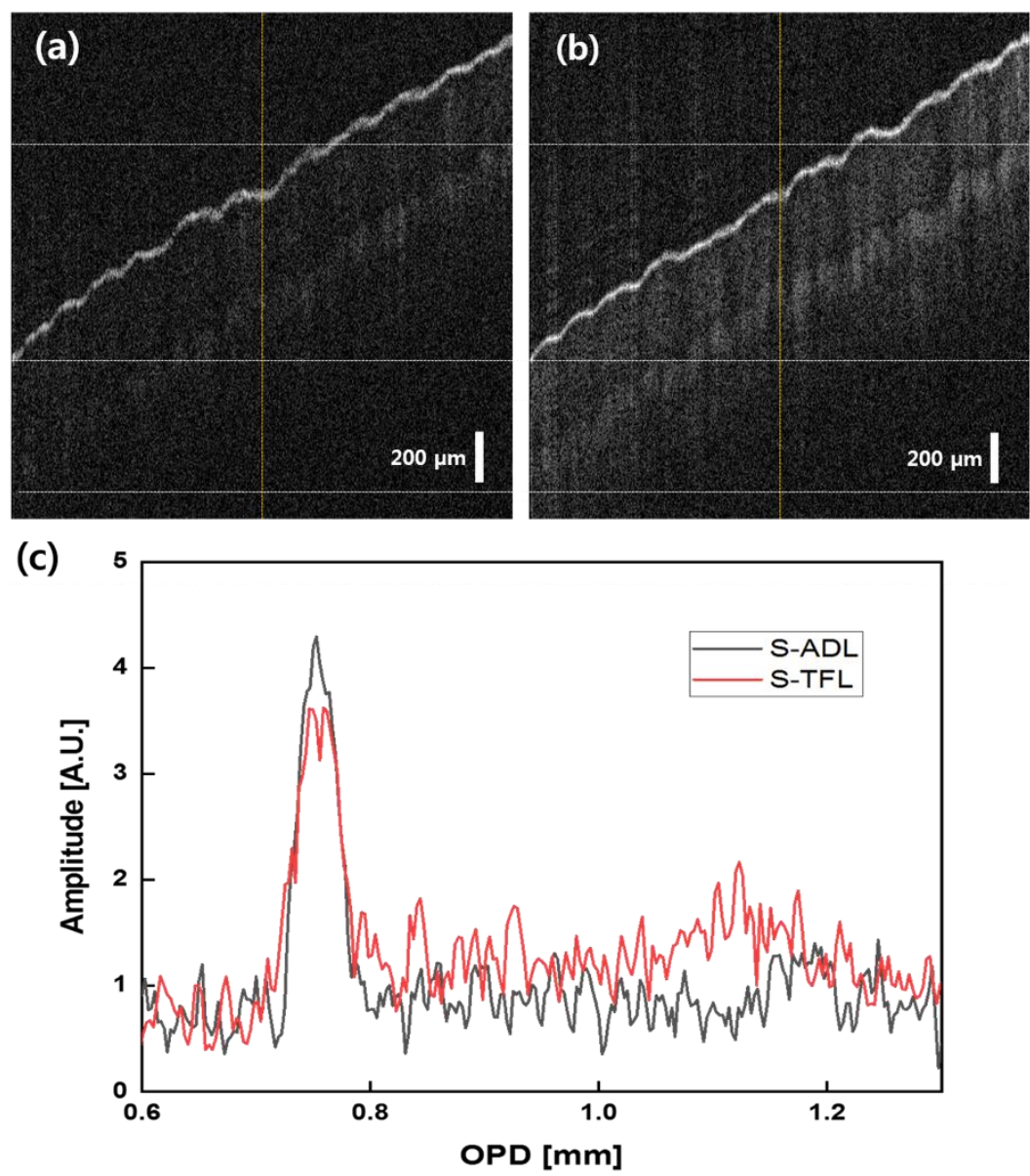

Figure 12. OCT images of the palm of a human hand obtained using (a) S-ADL and (b) S-TFL. (c) Depth profiles at the same A-line of each image.

\section{Conclusions}

In this paper, a method for improving SD-OCT performance based on a spectrometer using an aberration-corrected imaging lens is proposed. To do this, we designed a TFL with improved field curvature aberration characteristics. ADL was chosen as a reference for comparison because it is generally adopted in spectrometers in SD-OCT systems. The ADL used is a commercial product and the chromatic aberration is corrected well, but the field curvature aberration has not yet been addressed, thus the focal plane of ADL is not flat, but curved. These characteristics were observed in the optical simulation whereas the newly designed TFL exhibited an almost flat focal plane. The chromatic focal shift feature of the TFL was similar to the ADL with well-corrected chromatic aberration. The difference in the field curvature of the two imaging lenses was dramatic with regard to the change in spot size. The TFL showed an RMS spot radius of $5 \mu \mathrm{m}$ at the edge wavelength that matched the size of a single pixel whereas the ADL showed an RMS spot radius of $20 \mu \mathrm{m}$ at the same wavelength. The TFL showed better performance than the ADL in regard to MTF characteristics. Therefore, for a spectrometer with the ADL, the optical loss increases as the wavelength increases or decreases from the central wavelength. 
This results in reduced visibility of the spectral interference signal, which decreases the imaging depth that can be achieved. This fact well explains the experimental results for the degradation of axial resolution and the roll-off characteristics at deep depths. The S-FTL maintained an axial resolution of $7.8 \mu \mathrm{m}$ at $210 \mu \mathrm{m}$ even when the OPD increased, but when this axial resolution of $7.8 \mu \mathrm{m}$ was measured using the S-ADL, it increased to $12 \mu \mathrm{m}$ as the OPD increased to $2.5 \mathrm{~mm}$. According to the roll-off curve, the imaging depth at $3 \mathrm{~dB}$ was $0.75 \mathrm{~mm}$ for the S-ADL and $0.89 \mathrm{~mm}$ for the S-TFL, and the ranging depth at $6 \mathrm{~dB}$ was extended to $1.16 \mathrm{~mm}$ for the S-ADL and $1.33 \mathrm{~mm}$ for the S-TFL. The S-TFL showed better imaging depth that the S-ADL. SD-OCT images obtained for non-biological samples and biological samples, which were assessed by considering the differences in the performance of the spectrometers. In the image obtained with the S-TFL, the boundary was distinguishable even at a deeper depth due to the roll-off characteristic. As can be seen from the simulation results for aberration and spot size, the results obtained with the S-TFL were better than those obtained with the S-ADL regarding signal amplitude and contrast, and according to depth. The overall brightness of the SD-OCT images may be affected by the MTF characteristics. From the experimental results obtained with the spectrometers, we can see that the field curvature aberration correction of the spectrometer's imaging lens contributes significantly to improving the SD-OCT imaging performance.

Author Contributions: S.S.L. performed simulation on imaging lens aberration and implemented the spectrometer using the imaging lenses and SD-OCT system with the spectrometers. Also, S.S.L carried out all the experiments and signal processing for image reconstruction and contributed to the draft of the paper. W.S. conducted lens design with the help of the company and fabricated the imaging lens. W.S. also contributed to the draft of the paper. E.S.C. supervised the work including optical design, experiment, and data analysis and contributed to the draft of the paper. All authors have read and approved the final version of the manuscript.

Funding: This work was supported by the National Research Foundation of Korea (NRF) grant (NRF-2017R1A2B2009732 \& NRF-2015M3A9E2067140) funded by the Korea government (Ministry of Science, ICT, and Future Planning).

Acknowledgments: We would like to thank WINUS Technology Co., Ltd. for developing and manufacturing the lenses.

Conflicts of Interest: The authors declare no conflict of interest. The funders had no role in the design of the study; in the collection, analyses, or interpretation of data; in the writing of the manuscript, or in the decision to publish the results.

\section{References}

1. Huang, D.; Swanson, E.A.; Lin, C.P.; Schuman, J.S.; Stinson, W.G.; Chang, W.; Hee, M.R.; Flotte, T.; Gregory, K.; Puliafito, C.A.; et al. Optical coherence tomography. Science 1991, 254, 1178-1181. [CrossRef] [PubMed]

2. Kirby, M.; Ohtani, K.; Nickens, T.; Lisbona, R.M.L.; Lee, A.M.; Shaipanich, T.; Lane, P.; MacAulay, C.; Lam, S.; Coxson, H.O. Reproducibility of optical coherence tomography airway imaging. Biomed. Opt. Express 2015, 6, 4365-4377. [CrossRef] [PubMed]

3. Zuccaro, G.; Gladkova, N.; Vargo, J.; Feldchtein, F.; Zagaynova, E.; Conwell, D.; Falk, G.; Goldblum, G.; Dumot, J.; Ponsky, J.; et al. Optical coherence tomography of the esophagus and proximal stomach in health and disease. Am. J. Gastroenterol. Suppl. 2001, 96, 2633-2639. [CrossRef] [PubMed]

4. Bouma, B.E.; Villiger, M.; Otsuka, K.; Oh, W.Y. Intravascular optical coherence tomography. Biomed. Opt. Express 2017, 8, 2660-2686. [CrossRef]

5. Wang, H.; Kang, W.; Zhu, H.; MacLennan, G.; Rollins, A.M. Three-dimensional imaging of ureter with endoscopic optical coherence tomography. Urology 2011, 77, 1254-1258. [CrossRef]

6. Choi, W.J.; Wang, H.; Wang, R.K. Optical coherence tomography microangiography for monitoring the response of vascular perfusion to external pressure on human skin tissue. J. Biomed. Opt. 2014, 19, 056003. [CrossRef]

7. Morgner, U.; Drexler, W.; Kärtner, F.X.; Li, X.D.; Pitris, C.; Ippen, E.P.; Fujimoto, J.G. Spectroscopic optical coherence tomography. Opt. Lett. 2000, 25, 111-113. [CrossRef]

8. Pircher, M.; Goetzinger, E.; Leitgeb, R.; Hitzenberger, C.K. Three dimensional polarization sensitive OCT of human skin in vivo. Opt. Express 2004, 12, 3236-3244. [CrossRef] 
9. Schmitt, J.M. OCT elastography: Imaging microscopic deformation and strain of tissue. Opt. Express 1998, 3, 199-211. [CrossRef]

10. Gilgen, H.H.; Novak, R.P.; Salathe, R.P.; Hodel, W.; Beaud, P. Submillimeter optical reflectometry. J. Lightwave Technol. 1989, 7, 1225-1233. [CrossRef]

11. Kwong, K.F.; Yankelevich, D.; Chu, K.C.; Heritage, J.P.; Dienes, A. 400-Hz mechanical scanning optical delay line. Opt. Lett. 1993, 18, 558-560. [CrossRef] [PubMed]

12. Chinn, S.R.; Swanson, E.A.; Fujimoto, J.G. Optical coherence tomography using a frequency-tunable optical source. Opt. Lett. 1997, 22, 340-342. [CrossRef] [PubMed]

13. Yun, S.H.; Tearney, G.J.; de Boer, J.F.; Iftimia, N.; Bouma, B.E. High-speed optical frequency-domain imaging. Opt. Express 2003, 11, 2953-2963. [CrossRef] [PubMed]

14. Yun, S.H.; Boudoux, C.; Tearney, G.J.; Bouma, B.E. High-speed wavelength-swept semiconductor laser with a polygon-scanner-based wavelength filter. Opt. Lett. 2003, 28, 1981-1983. [CrossRef]

15. An, L.; Li, P.; Shen, T.T.; Wang, R. High speed spectral domain optical coherence tomography for retinal imaging at 500,000 A-lines per second. Biomed Opt. Express 2011, 2, 2770-2783. [CrossRef]

16. Golubovic, B.; Bouma, B.E.; Tearney, G.J.; Fujimoto, J.G. Optical frequency-domain reflectometry using rapid wavelength tuning of a Cr 4+: Forsterite laser. Opt. Lett. 1997, 22, 1704-1706. [CrossRef]

17. Lexer, F.C.K.H.; Hitzenberger, C.K.; Fercher, A.F.; Kulhavy, M. Wavelength-tuning interferometry of intraocular distances. Appl. Opt. 1997, 36, 6548-6553. [CrossRef]

18. Hausler, G.; Lindner, M.W. "Coherence radar" and" spectral radar"-new tools for dermatological diagnosis. J. Biomed. Opt. 1998, 3, 21-31. [CrossRef]

19. Klein, T.; Wieser, W.; Reznicek, L.; Neubauer, A.; Kampik, A.; Huber, R. Multi-MHz retinal OCT. Biomed. Opt. Express 2013, 4, 1890-1908. [CrossRef]

20. Xu, J.; Ishikawa, H.; Wollstein, G.; Kagemann, L.; Schuman, J.S. Alignment of 3-D optical coherence tomography scans to correct eye movement using a particle filtering. IEEE Trans. Med. Imaging 2012, 31, 1337-1345. [CrossRef]

21. Huang, Y.; Liu, X.; Kang, J.U. Real-time 3D and 4D Fourier domain Doppler optical coherence tomography based on dual graphics processing units. Biomed. Opt. Express 2012, 3, 2162-2174. [CrossRef] [PubMed]

22. Bonesi, M.; Minneman, M.P.; Ensher, J.; Zabihian, B.; Sattmann, H.; Boschert, P.; Drexler, W. Akinetic all-semiconductor programmable swept-source at $1550 \mathrm{~nm}$ and $1310 \mathrm{~nm}$ with centimeters coherence length. Opt. Express 2014, 22, 2632-2655. [CrossRef] [PubMed]

23. Ding, Z.; Ren, H.; Zhao, Y.; Nelson, J.S.; Chen, Z. High-resolution optical coherence tomography over a large depth range with an axicon lens. Opt. Lett. 2002, 27, 243-245. [CrossRef] [PubMed]

24. Lee, K.S.; Rolland, J.P. Bessel beam spectral-domain high-resolution optical coherence tomography with micro-optic axicon providing extended focusing range. Opt. Lett. 2008, 33, 1696-1698. [CrossRef]

25. Lorenser, D.; Singe, C.C.; Curatolo, A.; Sampson, D.D. Energy-efficient low-Fresnel-number Bessel beams and their application in optical coherence tomography. Opt. Lett. 2014, 39, 548-551. [CrossRef]

26. Yu, H.; Jang, J.; Lim, J.; Park, J.H.; Jang, W.; Kim, J.Y.; Park, Y. Depth-enhanced 2-D optical coherence tomography using complex wavefront shaping. Opt. Express 2014, 22, 7514-7523. [CrossRef]

27. Ravichandran, N.K.; Wijesinghe, R.E.; Shirazi, M.F.; Park, K.; Jeon, M.; Jung, W.; Kim, J. Depth enhancement in spectral domain optical coherence tomography using bidirectional imaging modality with a single spectrometer. J. Biomed. Opt. 2016, 21, 076005. [CrossRef]

28. Li, P.; An, L.; Lan, G.; Johnstone, M.; Malchow, D.S.; Wang, R.K. Extended imaging depth to $12 \mathrm{~mm}$ for 1050-nm spectral domain optical coherence tomography for imaging the whole anterior segment of the human eye at 120-kHz A-scan rate. J. Biomed. Opt. 2013, 18. [CrossRef]

29. Leitgeb, R.A.; Hitzenberger, C.K.; Fercher, A.F.; Bajraszewski, T. Phase-shifting algorithm to achieve high-speed long-depth-range probing by frequency-domain optical coherence tomography. Opt. Lett. 2003, 28, 2201-2203. [CrossRef]

30. Wang, Z.; Yuan, Z.; Wang, H.; Pan, Y. Increasing the imaging depth of spectral-domain OCT by using interpixel shift technique. Opt. Express 2006, 14, 7014-7023. [CrossRef]

31. Bajraszewski, T.; Wojtkowski, M.; Szkulmowski, M.; Szkulmowska, A.; Huber, R.; Kowalczyk, A. Improved spectral optical coherence tomography using optical frequency comb. Opt. Express 2008, 16, 4163-4176. [CrossRef] [PubMed] 
32. Hu, Z.; Rollins, A.M. Fourier domain optical coherence tomography with a linear-in-wavenumber spectrometer. Opt. Lett. 2007, 32, 3525-3527. [CrossRef] [PubMed]

33. Lan, G.; Li, G. Design of a k-space spectrometer for ultra-broad waveband spectral domain optical coherence tomography. Sci. Rep. 2017, 7, 42353. [CrossRef] [PubMed]

34. Moon, S.; Qu, Y.; Chen, Z. Characterization of spectral-domain OCT with autocorrelation interference response for axial resolution performance. Opt. express 2018, 26, 7253-7269. [CrossRef] [PubMed]

35. Wojtkowski, M.; Srinivasan, V.J.; Ko, T.H.; Fujimoto, J.G.; Kowalczyk, A.; Duker, J.S. Ultrahigh-resolution, high-speed, Fourier domain optical coherence tomography and methods for dispersion compensation. Opt. Express 2004, 12, 2404-2422. [CrossRef]

36. Lee, S.S.; Kwon, D.W.; Choi, E.S.; Song, W.S. Optical Design Method of a Spectrometer for Spectral Domain Optical Coherence Tomography. New Phys. Sae Mulli 2018, 68, 570-577. [CrossRef]

(C) 2020 by the authors. Licensee MDPI, Basel, Switzerland. This article is an open access article distributed under the terms and conditions of the Creative Commons Attribution (CC BY) license (http://creativecommons.org/licenses/by/4.0/). 